\title{
Deontología de la evaluación: el modelo de los códigos éticos anglosajones
}

María Bustėlo Ruesta *

\section{Introducción}

La consolidación profesional de una disciplina se puede medir a través de una serie de factores tales como el desarrollo de programas académicos superiores dedicados a la formación de profesionales (con todo lo que esto supone de elaboración de manuales, libros de texto, de lecturas y casos prácticos), la edición de publicaciones periódicas especializadas y la existencia de asociaciones profesionales. Otro factor, lógicamente, es la presencia de un mercado de trabajo con un cierto "volumen de negocion y la existencia de profesionales que son contratados como tales. Uno de los países en donde el grado de consolidación disciplinar del campo de la evaluación de programas y políticas es mayor es los Estados Unidos. Para ilustrar esta afirmación bastan algunos datos: la Asociación Americana de Evaluación tiene aproximadamente 3.000 socios y en sus conferencias anuales atrae una media de 1.000 profesionales; en un directorio reciente (ALTSCHULD y ENGLE, 1994) se identifican cuarenta y nueve programas especializados de formación académica y existen una serie de publicaciones periódicas en la materia con un alcance y una tirada importante ${ }^{1}$.

La elaboración de códigos de conducta o listados de principios éticos son también un indicador importante del grado de consolidación y madurez de una disciplina profesional. Estos códigos están normalmente vinculados a las asociaciones profesionales en las diferentes disciplinas. En los últimos años, se han ido elaborando diferentes códigos profesionales en el campo de la evaluación de programas y políicas, vinculados a las asociaciones profesionales de evaluadores que también han ido surgiendo, sobre todo, a partir de los años 80 (Joint Committee, 1981 y 1994; Asociación Americana de Evaluación, 1994; Asociación Canadiense de Evaluación, 1996; Asociación Australasiática de Evaluación, 1997).
En Estados Unidos existen actualmente dos códigos profesionales importantes y que son punto de referencia en el ámbito profesional y académico de la evaluación. Éstos son, por un lado, las Normas (Standards) elaboradas por Joint Committee on Standards for Educational Evaluation (1981, 1. e. edición y 1994, 2. ${ }^{a}$ edición) y, por otro, los Principios Orientadores para Evaluadores (Guiding Principles for Evaluators) de la Asociación Americana de Evaluación (adoptados y editados por la Asociación en 1994). De hecho, los otros dos códigos existentes en la actualidad en el campo de la evaluación son los que han sido elaborados por las respectivas asociaciones de evaluación canadiense y australiana, y que son posteriores a los códigos estadounidenses.

En este artículo se discute, en primer lugar, el significado e importancia de la deontología profesional en el campo de la evaluación que se ha desarrollado, principalmente, en comunidades científicas anglosajonas (Estados Unidos de América, Canadá y Australia) ${ }^{2}$. Posteriormente se analizan los códigos existentes en dicho campo, haciendo especial hincapié en los dos códigos generados en los Estados Unidos (las Normas del Joint Committee y los Principios Orientadores de la Asociación Americana de Evaluación), y comparándolos entre sí. Finalmente, se exponen algunas ideas para la atraducción de dichos códigos al contexto de evaluación español.

\section{El significado y trascendencia de la deontología profesional del evaluador}

Como ya se ha señalado anteriormente, la existencia de códigos deontológicos es, a la vez, un indicador y un elemento generador o provocador de una mayor consolidación institucio- 
nal y profesional de una disciplina. Profesiones fuertemente consolidadas desde hace tiempo (como son la medicina, la abogacía o la arquitectura) o más recientemente (como la psicología) han desarrollado este tipo de códigos. Además de fomentar un sentido de pertenencia e identificación entre los profesionales, acotan y regulan la práctica profesional entre sus respectivos campos.

Un aspecto de enorme trascendencia respecto a los códigos deontológicos es que permiten la introducción y desarrollo de los temas éticos, que resultan clave en la práctica de la evaluación. No se trata sólo de elaborar teóricamente sobre estos asuntos como parte intrínseca a la propia disciplina, sino también de orientar ante los conflictos y dilemas éticos que se van a producir, inevitablemente, en cualquier situación evaluativa. En cierto modo, se trata de afrontar esta realidad y de reconocer explícitamente el contexto político en el que está inmersa la evaluación. Este reconocimiento explícito se refleja en los códigos que se han elaborado. Por ejemplo, el Joint Commmittee, además de plantearse, con la elaboración de sus Normas, orientar el diseño, empleo y crítica de las evaluaciones y de animar a la utilización de una variedad de métodos en evaluación, pretende ayudar a los evaluadores a identificar y afrontar la realidad política. Textualmente este comité afirma que "las agendas políticas y el dinero son fuentes de poder que pueden corromper la evaluación en cualquier circunstancia. La adhesión a las Normas ayudará a minimizar las posibilidades de una mala utilización de ese poder por parte de los evaluadores o sus clientes" (Joint Committee, 1994, p. 4).

Sin embargo, se suelen formular los temas éticos con un alto grado de generalidad ${ }^{3}$. Esta generalidad, en cierto modo inevitable e incluso deseable para evitar la rigidez, es una de las mayores debilidades de este tipo de códigos. Una de las consecuencias de dicha generalidad tiene que ver con que estos códigos puedan aparecer como una forma de regular lo obvio. Ciertamente, los principios podrían ser aplicables a cualquier práctica profesional (especialmente los Principios Orientadores de la AEA) y están formulados de una forma muy general. Y esto tiene dos caras: por un lado, la necesidad y el afán de regular las conductas que tienen los anglosajones y que, desde una perspectiva más latina, nos puede producir sensaciones de extrañeza e incluso rechazo. Esta pasión por lo regulado hace que ya se esté empezando a hablar de acreditar evaluadores, siendo una cuestión candente en la actualidad en Estados Unidos ${ }^{4}$.

Por otro lado, la generalidad con la que están formulados los Principios también tiene que ver con el afán de ser comprehensivos, es decir, que todo el mundo pueda verse reflejado y no ofender a nadie, es decir, de ser políticamente correctos. Lo políticamente correcto en la actualidad en el campo de la evaluación consiste en ser flexible con las diferentes aproximacio- nes y modelos, tener en cuenta la vOz de los grupos minoritarios y aceptar las diferentes perspectivas, incluso aunque éstas sean diametralmente opuestas. De hecho, aunque la evaluación es uno de los campos en los que mejor han empezado a convivir las tradiciones cualitativa y cuantitativa, ya que muchos evaluadores utilizan una mezcla de métodos y técnicas, sigue habiendo evaluadores cuantitativistas y cualitativistas que hablan lenguajes distintos. Este intento de integración de todas las posibles tendencias o, mejor dicho, el miedo a ser políticamente incorrectos, ha hecho que queden unos Principios un tanto insustanciales o desabridos, dejando las orientaciones quizá demasiado abiertas. De hecho, alguna de las críticas de los Principios Orientadores de la AEA van en esta dirección. Peter $\mathrm{H}$. Rossi (Rossi, 1995) coincide con CHELImsky en que los Principios Orientadores de la AEA no se preocupan de forma especial por la integridad de los resultados ni en hacer al evaluador lo suficientemente valiente a la hora de presentar sus resultados que, según Rossi, están "avalados por la validez de su investigación". En su crítica feroz a los Principios, desde una perspectiva netamente positivista $y$, por lo tanto, valiente para los tiempos que corren (es posible que hablar de La Verdad con mayúscula empiece a ser considerado políticamente incorrecto), llega a decir que la evaluación "no es una negociación para conseguir aprobación sino la búsqueda de la mejor aproximación a la verdad" (Rossi, 1995, p. 58). Rossi considera que los Principios de la AEA son débiles y que "... los códigos potentes y detallados existen en los campos profesionales en los que se ha alcanzado consenso sobre la sustancia y el método, mientras que los códigos débiles afloran en las asociaciones en las que los miembros están divididos en dichas materias" (RossI, 1995, p. 55).

Finalmente, esta generalidad también está relacionada con la idea de no "sentar cátedra" cuando se trata de valores, especcialmente cuando se viene de una tradición en las ciencias sociales en la que se ha pretendido, durante muchos años, que los valores tuvieran muy poco que ver con la práctica científica (House, 1994). En este sentido, uno de los Principios Orientadores de la $\mathrm{AEA}$ es especialmente criticado por general: el referido a las "responsabilidades hacia el bienestar público y general. Como se verá más adelante, esta generalidad a la que nos referimos está menos presente en las Normas del Joint Committee y en las orientaciones éticas de la Sociedad Australasiática de Evaluación y más presente en las orientaciones de las asociaciones americana y canadiense.

En la introducción de este apartado se señalaba la importancia de los códigos deontológicos para la creación de un sentido de pertenencia e identificación a una determinada comunidad profesional. Esta función de la deontología profesional nos parece clave y además es reconocida por parte de las organizaciones que han generado los códigos en el campo de la evaluación. De este modo, la AEA considera abierta y explícitamente sus Princi- 
pios Orientadores como un medio para la socialización de sus miembros. Más allá, no sólo se pretende que los Principios Orientadores sean una buena excusa para la discusión entre profesionales, sino que también sean un catalizador para la identificación de los profesionales como tales; es decir, que se genere grupo y sentido de pertenencia a una profesión que ha ido adquiriendo definición, autonomía y potencia creciente en los últimos 25 ó 30 años.

Otra idea subyacente al desarrollo de estos códigos es la idea del profesional de la evaluación como especialista específico en el campo de diseño y metodología de evaluación, en oposición a la idea de los especialistas en la materia de la que se ocupa el programa evaluado. Ésta ha sido una polémica recurrente durante años y, a su solución, contribuye, de forma clara, una mayor consolidación institucional del campo profesional de la evaluación. Los códigos se decantan, claramente, por profesionales especialistas en evaluación que posteriormente pueden especializarse en la evaluación de determinadas políticas o programas, aunque también está implícita la idea de no "encasillarse" excesivamente en sólo un campo o área para no perder la orientación inicial de la evaluación y para tener un "registro" más amplio como evaluadores. Esto a veces es difícil porque, efectivamente, las personas que evalúan una política y programa en un determinado ámbito adquieren un alto grado de conocimiento sobre el programa o política evaluada, y tienen grandes probabilidades de acabar teniendo una posición privilegiada en el correspondiente policy network. Aunque las existencia de asociaciones profesionales y publicaciones especializadas está contribuyendo a formar un perfil de evaluadores especializados, sigue siendo común —especialmente en los países en los que el grado de consolidación institucional de la evaluación es bajo, como es el caso español- que se contrate para realizar evaluaciones a especialistas en la materia y no en evaluación y que la evaluación siga siendo una excusa para hablar de la materia que se evalúa obviando el planteamiento y la función, el diseño, la metodología y las consecuencias de los procesos evaluativos. Así, es común encontrar escritos cuyo título incluye el concepto de evaluación pero en cuyo contenido se obvia (por ejemplo "Evaluación de la política de juventud" para hablar sobre política de juventud y no sobre cómo se ha evaluado dicha política).

Esta cuestión de quiénes deben ser los profesionales de la evaluación es, en cierto modo, compleja, ya que la evaluación es una especialización de las ciencias sociales. Es decir, en los países en los que el campo profesional está más consolidado, la especialización de evaluación se adquiere en programas de postgrado (o tercer ciclo académico) y requiere una formación previa e inicial en alguna de las ciencias sociales (ya sea educación, psicología, sociología, ciençia política, economía, trabajo social, etc.). Esta formación previa puede condicionar, de algún modo, los sectores en los que trabajen los futuros evaluadores (así, los evaluadores pedagogos suelen evaluar programas y políticas de corte educativo, los psicólogos de promoción de la salud, los politólogos programas de modernización administrativa, etc.), pero no tiene que ser necesariamente de este modo, debido a la naturaleza multi e interdisciplinar de la evaluación. En países como España, en donde existe una cierta tendencia hacia el corporativismo profesional, habrá que tener cierta cautela para que la evaluación no se convierta en una disciplina exclusiva de un determinado campo profesional, o para que la evaluación se desarrolle separada y diferenciadamente en los distintos campos profesionales, perdiendo su enriquecedor potencial como campo multidisciplinar. En la actualidad, las asociaciones de evaluación acogen a una variedad de profesionales, reflejando dicho carácter multidisciplinar.

Relacionado con lo anterior, otro papel importante que cumplen los códigos tiene relación con la formación de futuros evaluadores, ya que sirven como una guía estructurada sobre el perfil del buen evaluador. En el caso de las Normas del Joint Committee son una guía no sóló para los evaluadores sino para los procesos de evaluación en general. Siguiendo dichas Normas se tiene una visión completa y estructurada de cómo debe hacerse una evaluación. Esto resulta una fuente de especial utilidad para la formación de académicos y profesionales en el campo de la evaluación. Las Normas, además, están editadas en un formato de carácter didáctico con casos y ejemplos ilustrativos y con una estructura sumamente útil para propósitos formativos. Los Principios Orientadores son también útiles como documento para el debate y la discusión en los programas de formación en evaluación.

Esta consideración del evaluador profesional como principal leit-motiv de los códigos deontológicos que supone una serie de fortalezas ya discutidas puede derivar también en una debilidad. Este tipo de códigos enfatiza demasiado la figura del evaluador, pudiendo dar una cierta impresión de omnipotencia del mismo. Es decir, las situaciones evaluativas son controlables, principalmente, por el evaluador. Y esto es, en muchísimos casos, falso o, cuanto menos, ingenuo. En su crítica a los Principios Orientadores de la AEA, Eleanor CHELIMSKY (CHelimsky, 1994) opina que se ha prestado mucha atención a los fallos del evaluador pero no tanta a las dificultades inherentes al contexto en el que se genera la evaluación. Y el contexto hace que los evaluadores deban ser especialmente valientes para realizar las evaluaciones tal y como ellos creen que deben hacerse. En definitiva, se trataría de enfatizar una mayor independencia del evaluador, teniendo en cuenta que no siempre el contexto es controlable por el mismo.

Finalmente, la deontología profesional en el campo de la evaluación puede cumplir otro importante papel para el desarrollo de la disciplina. Al igual que los códigos pueden ser úti- 
les para la tormación de evaluadores, también pueden serlo respecto a la formación de clientes, gestores y supervisores de evaluación. En definitiva, para quienes deciden sobre qué evaluaciones hay que realizar y formulan las políticas de evaluación. Precisamente porque estas personas, en muchos casos no tienen formación específica en evaluación -especialmente en nuestro país, ya que hay muy pocas personas con formación especializada en este ámbito-, es crucial la función de orientación a la que pueden servir no sólo respecto a los procesos de evaluación en sí mismos sino también respecto a qué conducta esperar de los evaluadores. Así, los códigos pueden ser un buen instrumento que propicie la discusión y facilite la comunicación entre gestores, clientes y equipos evaluadores. Especialmente, si en la organización o departamento que encarga la evaluación o en la que se gestiona el programa evaluado no hay cultura evaluativa, disponer de un documento de este tipo puede ayudar a los evaluadores a tratar temas importantes con sus clientes con una mayor legitimidad.

\section{Los códigos y su proceso de elaboración}

En Estados Unidos, el campo que actúa como pionero en el desarrollo deontológico relacionado con la evaluación es el educativo y, en cierto modo, el psicológico. Esto se explica por dos razones. La primera tiene que ver con el hecho general de que la evaluación de programas nace de manos de pedagogos que parten de la evaluación del alumno para trasladarla a la evaluación de las acciones y programas educativos (se puede hablar de Ralph TyLER en los años 40 y, en su aplicación más concreta, de Lee CRONBACH en los 60). Es decir, los primeros años de desarrollo de la disciplina se realizan, con especial fuerza, en el ámbito de la evaluación educativa. En segundo lugar, durante los años 60 y 70 existe, en Estados Unidos, un fuerte desarrollo de tests psicológicos que se aplican de forma sistemática a lo largo del sistema educativo y una especial obsesión por la medición de factores psicológicos y educativos en la población escolar. De esta manera, se comienzan a desarrollar normas y códigos deontológicos que regulen, de alguna manera, la aplicación de dichos procedimientos estandarizados. Así, las primeras normas relacionadas con la evaluación de programas (las del Joint Committee) se comienzan a desarrollar a partir de la iniciativa de un comité que estaba revisando normas para la aplicación y uso de manuales y tests psicológicos y educativos y que recomienda el desarrollo de unas normas específicas en el campo de la evaluación '.
Así, en el ano 1975 un comité nombrado por la Asociación Americana de Investigación Educativa (AERA), la Asociación Americana de Psicología (APA) y el Consejo Nacional para la Medición Educativa (National Council on Measurement in Education) comienza a reunirse para elaborar unas normas en el campo de la evaluación educativa. Se decide extender la invitación a otras nueve organizaciones y se crea así el Joint Committee on Standards on Educational Evaluation, que comienza a trabajar en el otoño de aquel año. Hasta la publicación de la primera edición en 1981, el Comité desarrolla un proceso público sistemático para establecer y probar las Normas y en el que participan personas expertas, usuarios y clientes de evaluaciones, etc. Estas colaboraciones (más de 200 personas expertas participan en dicho proceso) se realizan a través de un complejo entramado de paneles de escritores, paneles de revisión, trabajo de campo para la comprobación de las Normas, audiencias públicas, paneles de validación, etc. En el mismo año que se publica la primera edición de las Normas, el Joint Committee se establece como una organización no lucrativa dedicada al desarrollo de Normas de evaluación, su revisión periódica, su apoyo y promoción de su uso, además de la investigación y desarrollo relacionada con dichas Normas.

La primera edición de las Normas tiene tal impacto en el campo de la evaluación, tanto en Estados Unidos como internacionalmente, que trasciende el campo educativo alrededor del cual se habían gestado y se convierten en referencia general para la evaluación de programas, independientemente del ámbito de especialización. Esto hace que la revisión que comienza en 1989 y que dará origen a la segunda edición de las Normas, se plantee, sobre todo, con la intención de ampliar el campo de aplicación. Sin abandonar del todo el campo educativo (como ya se ha comentado, es la disciplina "madre". de la evaluación de programas y una de las que más ha aportado a la profesión en Estados Unidos), la revisión se realiza con una visión más amplia, incluyendo expertos en diferentes campos y probando las Normas en muy diferentes situaciones y programas. El resultado de la revisión, como se verá más adelante, no es un cambio sustancial, pero sí se incluyen ejemplos no sólo en el terreno educativo (colegios, universidades), sino también en el campo sanitario y de la salud, de la empresa, de los servicios sociales y del gobierno y la administración pública. El Joint Committee sigue desarrollando, probando y revisando las Normas en la actualidad. De hecho, en la segunda edición de las Normas hay un apartado especial en el que se anima a todas las personas que se dedican a la evaluación y utilizan las Normas a compartir sus experiencias. Para esto, el Joint Committee ha preparado un paquete de información que contiene una carta de reconocimiento, información sobre el proceso de revisión y actualización y una serie de hojas de retroalimentación que se pueden encargar directamente a la organización. A cambio, el evaluador debe describir su papel y responsabilidades en el 
proceso de evaluación, preparar un resumen de la evaluación incluyendo la aplicación de las Normas, mandar copia de los informes y describir ios problemas y conflictos surgidos en la aplicación de cada una de las Normas, así como la forma de solucionarlos. Por último, se le pide que sugiera posibles mejoras e identificar áreas no cubiertas por las Normas.

El segundo código al que se hace referencia son los aPrincipios Orientadores para evaluadores" (Guiding Principles for. Evaluators) desarrollados y aprobados por la Asociación Americana de Evaluación (AEA). Esta asociación fue creada en el año 1986, fundiendo así otras dos asociaciones existentes hasta entonces (la Evaluation Research Society -ERS- y el Evaluation Network). Aunque la ERS había elaborado unas normas publicadas en 1982, éstas ni habían adquirido tanta trascendencia como las del Joint Committee ni habían sido oficialmente adoptadas por la AEA. Así, en 1992, la AEA nombra una comisión para el desarrollo de estos Principios. Dicha comisión, integrada por William SHadish, Dianna Newman, Mary Ann ScheiRER y Christopher WYE, consideró todos los códigos relevantes de otras organizaciones profesionales y los miembros de la comisión aportaron algunos trabajos previos en temas éticos: SHADISH y NEWMAN, desde un punto de vista académico, sus escritos sobre la teoría del valor y sobre normas y principios de evaluación respectivamente, SCHEIRER desde la práctica privada como evaluadora de programas federales y WYE, desde la Academia Nacional de Administración Pública, en la que había estado trabajando en las dimensiones éticas del servicio público y los administradores públicos. Cada miembro escribió un borrador independiente que, posteriormente, fue fundido en un documento que se envió a todos los miembros de la asociación con la idea de obtener respuesta de los mismos. La versión final se aprueba por el Consejo de Administración de la AEA en enero de 1994 y por sus miembros en el verano del mismo año. A partir de su aprobación y publicación, los Principios Orientadores se han convertido en un punto de referencia en muchos escritos sobre evaluación en Estados Unidos y se ha comenzado a desarrollar un trabajo intenso relacionado con los temas éticos en evaluación. Un indicador de este desarrollo es la reciente creación de una sección sobre "Retos Éticos" en la la revista de la Asociación Americana de Evaluación (recientemente renombrada The American Journal of Evaluation, anteriormente Evaluation Practice). En esta sección se presentan situaciones o supuestos en los que existe un cierto dilema ético y se pide a académicos o profesionales renombrados en evaluación que hagan un comentario sobre los mismos y den su opinión sobre cómo ellos resolverían dicho dilema o situación; para esto se pide que se haga especial referencia a los Principios Orientadores de la AEA y a las Normas del Joint Committee. Como anécdota que muestra la importancia concedida a estos Principios Orientadores como referente esencial en la formación de evaluadores, se puede mencionar un artículo en el que la autora propone una regla nemotécnica relacionada con el cuento de "El Mago de Oz" para que los estudiantes de evaluación puedan recordar más fácilmente los cinco Principios Orientadores ${ }^{6}$. En cualquier caso, la publicación de los Principios Orientadores ha sido seguida en estos últimos años de un trabajo serio y constante sobre los mismos; de hecho, en los propios Principios Orientadores, en las consideraciones previas, se indica que los Principios Orientadores deberán de ser revisados al menos cada cinco años.

Los otros dos códigos que se contemplan en este artículo, los elaborados por la Sociedad Canadiense de Evaluación y por la Sociedad Australasiana de Evaluación, son mucho más recientes, siendo aprobados, en 1996 y 1997 respectivamente. En ambos casos reciben el título de "Orientaciones para una Actuación Ética" (Guidelines for Etbical Conduct), aunque su estructura es diferente (ver anexo).

El proceso de elaboración del código canadiense comienza con un pequeño comité que estudia los códigos existentes en otras asociaciones relacionadas y prepara un borrador que se revisa por parte del Consejo de la asociación. Un segundo borrador se distribuye entre los miembros para sondear comentarios y sugerencias y se discute en la conferencia anual de la sociedad. Un tercer borrador se vuelve a distribuir entre los miembros a través del boletín de la asociación, recogiendo sus aportaciones. La versión última se aprueba en la conferencia anual de mayo de 1996.

En el caso de Australasia, el Consejo de Dirección de la AES organiza un comité para la elaboración del código en agosto de 1996. Dicho comité establece una ronda de consultas con los miembros de la asociación a través de los grupos regionales a lo largo del año 1997. Las Orientaciones se aprueban por el Consejo de Dirección en diciembre de 1997. Estas Orientaciones se complementan con las Normas del Joint Committee que fueron asumidas oficialmente por el Consejo de la AES en agosto de 1996 y con un Código Ético Provisional que se está revisando a lo largo de 1998 y que se centra en el comportamiento de los evaluadores y se basa en principios éticos similares a los que subyacen a las Orientaciones.

\section{Los criterios deontológicos a partir de la comparación entre los códigos}

En este apartado se discuten los criterios deontológicos a partir de los códigos existentes en el campo de la evaluación. Se hace especial referencia, en la primera parte, a los dos códi- 
gos estadounidenses ya que éstos son los que primero se elaboran $y$, por lo tanto, tienen una mayor trascendencia, incluso como referencia para los que se realizan posteriormente (canadiense y australiano). Al final de este apartado, se describen brevemente estos otros dos códigos y se apuntan diferencias generales con los del Joint Committee y la AEA.

Los dos códigos estadounidenses son muy diferentes, aunque ambos son referencia obligada en el campo de la evaluación, especialmente en el ámbito anglosajón. En este escrito se discuten los criterios deontológicos según los propósitos diferenciales que han generado el establecimiento y desarrollo de los dos códigos, el ámbito de aplicación de cada uno, la estructura de los mismos, así como su antigüedad y momento de formulación y revisión.

\section{Finalidad de las normas de ética profesional}

Respecto a los propósitos que generan los códigos éticos profesionales, es evidente que existe un intento subyacente de institucionalización del campo profesional y académico del que se trate, en este caso, de la evaluación. Sin embargo, se pueden encontrar matices distintos en los dos códigos analizados.

En el caso del Joint Committee, ya hemos visto que dicho comité fue creado como una prolongación de otro comité que establecía normas para la utilización de test educativos y psicológicos y su primer propósito consistía en desarrollar Normas para la evaluación educativa en general y no sólo para la utilización de tests en evaluación. Dicho proceso de elaboración se hizo cuidadosa y concienzudamente e implicando a diferentes asociaciones profesionales y a un número significativo de profesionales y académicos de la evaluación. Inmediatamente después de la publicación de la primera edición de las Normas -que se hace después de cinco años de proceso de elaboración- el Joint Committee se convierte en una organización autónoma y con vida propia cuyo objetivo es el desarrollo y revisión de dichas Normas. En su segunda edición el Joint Committee expresa que las Normas son un esfuerzo de ofrecer orientación para realizar evaluaciones efectivas y que "tomadas en su conjunto, las 30 Normas ofrecen una filosofía de trabajo para la evaluación" (Joint Committee, 1994, p. xviii). El comité deja claro que las Normas en sí mismas no garantizan una buena evaluación y que se requiere del buen juicio profesional para aplicar adecuadamente dichas Normas a cada una de las situaciones evaluativas. También es consciente de que las Normas deberán ir evolucionando a medida que se desarrolle y evolucione el campo profesional de la evaluación, pero también de que la existencia misma de las Normas contribuye significativamente a la mejora de la evaluación.
En lo que se refiere a los Principios Orientadores de la AEA, esta asociación se planteó la necesidad de articular unas normas o principios propios, distintos de los del Joint Committee. Las dos razones esgrimidas entonces fueron, por un lado, el hecho de ser la principal organización profesional de evaluadores en EE.UU. y que, por lo tanto, no se debía depender de normas establecidas por otra organización, sino por unas propias. Por otro lado, se consideraba que el Joint Committee había crecido al amparo, sobre todo, de la evaluación educativa, y que tenía que haber un código que reflejara otras áreas y el carácter multidisciplinar de la evaluación. Tanto la AEA como la comisión designada para la elaboración del código tienen claro, desde un principio, que se quieren establecer principios generales y orientadores más que normas específicas. Ésta es ya una importante diferencia con las Normas, ya que los Principios están pensados para ser más conceptuales que operativos, ofreciendo orientación a nivel general más que sobre cómo tomar decisiones específicas de tipo metodológico y táctico. Las razones para centrarse en principios generales las explican los miembros de la comisión (SHADISH et al., 1995, p. 4): en primer lugar, los principios son un prerrequisito de las normas; segundo; el desarrollo de principios es más rápido que el de normas y, finalmente, así se evitaría el posible solapamiento con las Normas del Joint Committee.

Como es lógico en cualquier actividad promovida por una asociación profesional, el establecimiento de Principios Orientadores por parte de la AEA tiene como finalidad contribuir a una mayor institucionalización de la profesión. Además, también se plantean, explícitamente, promover la socialización de sus miembros; es decir, propiciar la discusión y el intercambio de los mismos así como fomentar su identificación "como grupo y con el campo profesional. Aunque ésta no haya sido una finalidad explícita del Joint Committee, ha cumplido un papel importante al respecto.

Resumiendo respecto a los propósitos de los dos códigos, mientras los Principios de la AEA pretenden una orientación más general que va más allá incluso de la tarea específica de la evaluación (promueven un estilo de vida profesional que podría ser aplicable a otras profesiones), el foco de las Normas del Joint Committee se encuentra en la evaluación en particular y en su solidez como tal.

\section{El ámbito de aplicación de los códigos deontológicos}

También podemos encontrar algunas diferencias respecto al ámbito de aplicación de ambos códigos. A continuación, se tratan los campos profesionales de aplicación, 
el tipo de evaluación que se promueve y los destinatarios a los que se pretende llegar.

Ya se ha visto que el Joint Committee se forma' en el ámbito de la evaluación educativa, mientras la AEA tiene por objeto la evaluación en todos los campos profesionales posibles. El Joint Committee decide centrarse en su primera edición en la evaluación de programas, proyectos y materiales, dejando fuera tanto la evaluación institucional como la evaluación de personal (de hecho, el propio Comité elabora aparte The Personnel Evaluation Standards en 1988, que no tendrán tanta trascendencia en el campo de la evaluación). Como ya se ha comeñtado, la revisión de las Normas y su consecuente segunda edición de 1994 viene en cierto modo determinada por el creciente interés de otros evaluadores en otros campos de aplicar las Normas a sus respectivos ámbitos, $y$, de hecho, éstas se aceptan y son referencia en el campo de la evaluación en general. Sin embargo, su leitmotiv inicial sigue siendo el campo de la evaluación educativa.

Los Principios Orientadores de la AEA se elaboran, sin embargo, para todo tipo de evaluación, incluyendo la evaluación de programas, proyectos, materiales, productos, personal, políticas (que en EE.UU. se suele entender como la evaluación de las consecuencias de la implantación de medidas públicas), desempeño, tecnología, investigación, teoría, y de la propia evaluación (metaevaluación). En cualquier caso, uno y otro código parecen tener como referencia principal la evaluación de programas y políticas en sentido amplio. Eso sí, escorados ambos hacia situaciones de evaluación externa en la que una organización —en la mayoría de los casos distinta a la que ejecuta el programa y en muchos incluso a la que lo financia- financia la evaluación y contrata a una consultora o a un evaluador o equipo evaluador independiente). Ésta ha sido una de las mayores críticas que se han recibido, sobre todo, desde una perspectiva externa a los Estados Unidos (HENDRicks y ConNer, 1995).

Otro aspecto interesante a tener en cuenta respecto al ámbito de aplicación es la comparación de los principales destinatarios de ambos códigos. Mientras las Normas del Joint Committee se concentran más en la situación evaluativa en general y tienen en cuenta, así, tanto evaluadores como clientes de la evaluación (en el sentido de quiénes encargan las evaluaciones), los Principios de la AEA parecen estar especialmente pensados para los evaluadores. Esto puede parecer normal en el sentido de que nacen de una asociación de profesionales de la evaluación; sin embargo, conlleva una cierta contradicción ya que en dicha asociación se ha promovido la entrada y desarrollo profesional del cliente de la evaluación (existe una comisión de trabajo integrada por clientes de evaluaciones y en las conferencias anuales suele haber mesas y paneles desde ese punto de vista). Al fin y al cabo, considerar a los contratadores (encargadoress) y supervisores de las evaluaciones también como profesionales de la evaluación es, de alguna forma, otro indicador del grado de institucionalización del campo profesional.

\section{La estructura de los códigos}

En lo que se refiere a la estructura de los códigos, también se pueden encontrar diferencias. En el caso de las Normas del Joint Committee, éstas se estructuran en cuatro factores: utilidad, factibilidad, corrección o legitimidad y precisión. En total son 30 Normas (ver Anexo 1). Los Principios de la AEA son tan sólo cinco principios generales (indagación sistemática, competencia, integridad/honestidad, respeto por las personas y responsabilidades hacia el bienestar público y general), aunque cada uno de ellos se ilustra con una serie de aclaraciones que profundizan en el significado de los Principios y orientan sobre su aplicación. Hay un total de 23 aclaraciones. Es decir, la estructura no es tan dispar como en un principio pueda parecer.

Aquí puede resultar interesante hacer un alto para examinar cuáles han sido los cambios de la 1. ${ }^{\text {a }}$ (1981) a la 2. edición (1994) de las Normas del Joint Committee. Realmente, se puede concluir que las diferencias son de matiz entre una edición a otra, aunque resulta interesante analizar la dirección de dichos cambios. Entre otras cosas, demuestra, en cierto modo, cuál ha sido el cambio en el campo profesional de la evaluación en los últimos años. Principalmente las diferencias tienen que ver con una visión más amplia y tolerante de la evaluación y las ciencias sociales en general. En los cambios de 1994 se deja entrever la admisión de diferentes perspectivas, mientras que en 1981 se utilizaba un lenguaje muy. dominado por el paradigma positivista. Así, los términos utilizados en la primera edición como información fiable, "medición válida y fiable", "control sistemático de datos e unforme objetivo se sustituyen por los términos "información defendible, "información válida y fiable", "información sistemática" e "informe imparcial" en la edición de 1994. Hay algunas Normas nuevas en la 2. a edición, como son la de metaevaluación y la orientación de servicio y algunas son más realistas y/o más apropiadas en su formulación ("documentación del programa" en lugar de "identificación del objeto" o revelación de resultados" en lugar de "derecho público a la información"). 
Finalmente, algunos pares de Normas antiguas se funden en una única ("oportunidad y difusión del informe" y "valoración completa y justa" antes "exposición total y franca" $y$ "equilibrio del informe").

También resulta interesante comparar la estructura de ambos documentos. En el caso de las Normas del Joint Committee, por igual en las dos ediciones, encontramos una visión general que incluye definiciones de los términos más importantes en evaluación y una exposición de las Normas absolutamente didáctica: resumen de las Normas de cada tipo (utilidad, factibilidad, etc.) y, por cada norma, además de su definición, una explicación general de la misma, orientaciones para su aplicación, un listado de los errores más comunes que suelen ocurrir al respecto y uno o dos casos ilustrativos con su correspondiente descripción y análisis. Finalmente, se ofrece, por cada norma, documentación y bibliografía de apoyo. También resulta interesante, y especialmente útil, el índice funcional de las Normas, apareciendo el listado de las Normas más relevantes a tener en cuenta para cada fase o tarea de la evaluación: la decisión de evaluar o no, la definición del problema de evaluación, el diseño de la evaluación, la recopilación de información, el análisis, el informe y la comunicación de resultados, la presupuestación de la evaluación, la contratación de la evaluación, la gestión de la evaluación y el reclutamiento de personal para la evaluación.

Los Principios Orientadores de la AEA, sin embargo, aparecen en un formato menos acabado, aunque el documento contiene, además de los Principios y sus aclaraciones como ya hemos comentado, dos secciones previas muy interesantes. La primera sección introductoria explica el origen y proceso de elaboración de los principios, además de una recomendación explícita de trabajo continuado. Una segunda sección, a modo de prefacio, expone diez premisas ${ }^{7}$ relacionadas con el desarrollo de los Principios.

4. Momento de elaboración de los códigos y antigüedad de los mismos

Finalmente, resulta evidente que sí existe una diferencia respecto a la antigüedad de ambos códigos. Aunque la segunda edición de las Normas es del mismo año que los Principios de la AEA, se nota que existe todo un trabajo previo que tiene ya más de 20 años en el caso del Joint Committe. Hay que tener en cuenta, además, que en dicho comité han participado un gran número de expertos. En el caso de los Principios de la AEA se elaboran por parte de un comité de cuatro personas $y$, aunque se dan a conocer y revisar por parte de todos los miembros de la AEA, dicho proceso no estaba tan estructurado como en el caso del Joint Committee.

En general y como conclusión de este análisis comparativo, aunque con las diferencias señaladas, los dos códigos tratados coexisten sin contradicciones, cumpliendo cada uno su papel. SANDERS (1995, pp. 48-49) realiza una comparación entre los dos códigos y llega a la conclusión de que no existen conflictos o inconsistencias entre ambos documentos. Los dos códigos enfatizan la precisión de resultados, la inclusión de los diferentes implicados en el proceso de evaluación y se preocupan tanto del bienestar de los participantes en la evaluación como de ofrecer un servicio a los implicados en el objeto evaluado, la comunidad y la sociedad en general. De hecho, cada uno de los códigos ofrece orientación en aspectos distintos de la conducta profesional: los Principios de la AEA en los valores profesionales y las Normas del Joint Committee en el desempeño profesional.

\section{Las diferencias con los códigos canadiense y australasiático}

Como se puede observar en el Anexo 1, de entre los cuatro códigos examinados, el canadiense es el más sencillo, con tres grandes apartados (competencia, integridad y responsabilidad) y once orientaciones. Las 22 orientaciones australianas están organizadas según las fases de la evaluación: encargo y preparación de la evaluación (dos principios generales y 9 orientaciones), realizacion de la evaluación (dos principios y 8 orientaciones) e información sobre los resultados de la evaluación (un principio y 5 orientaciones). En ambos casos se dirigen a la evaluación de programas en sentido amplio, bien sean internas o externas, grandes o pequeñas. Su propósito tiene que ver con servir de orientación para un comportamiento evaluativo ético y para ayudar en la toma de decisiones sobre la evaluación, así como asistir a los evaluadores en la resolución de los dilemas o conflictos éticos que se les puedan presentar en su práctica profesional. En general, se puede decir que, aunque están en una primera fase de desarrollo, ambos códigos pertenecen a una "segunda generación" de códigos profesionales y que ven la luz con un formato más "ordenado" que, por ejemplo, el de la AEA. Es indudable que estos últimos códigos se han beneficiado de las primeras experiencias estadounidenses.

Si se observa la tendencia creciente a la creación de asociaciones profesionales de evaluación y su consiguiente elaboración y aprobación de los respectivos códigos deontológicos, es probable que la European Evaluation Society, de reciente creación (fundada en 1994 y funcionando como tal desde enero de 1996) se plantee próximamente algún trabajo relacionado con la ética en evaluación y que considere, incluso, la elaboración de un código propio. 


\section{Conclusiones desde una perspectiva española}

En España existe un todavía pequeño pero incipiente campo profesional y académico de evaluación, sobre todo en lo referente a las políticas y los programas públicos. Como ya se ha mencionado anteriormente, el grado de consolidación profesional, institucional y académica de dicho campo es, hoy por hoy, muy bajo. No existe una asociación profesional a nivel nacional aunque algunas instituciones y miembros individuales pertenecen a la recientemente creada Sociedad Europea de Evaluación. Tampoco existen publicaciones cientificas periódicas específicas, aunque algunas empiezan a demostrar su claro interés por este campo (un ejemplo claro es este número monográfico). Para este campo incipiente puede resultar de especial utilidad contar con este tipo de códigos que, aunque haya que traducirlos al contexto español y en algunas ocasiones estén ufuera de tono" o no sean aplicables, sirven como documentos que contribuyen al acervo bibliográfico específico sobre evaluación, pueden provocar la reflexión y sirven como referencia para la institucionalización del campo en nuestro país.

Es cierto que todavía nos queda mucho para pensar en una producción propia de códigos de este tipo. También hay que reconocer que la aplicación de estos códigos, hoy por hoy, no es casi nunca posible en nuestro contexto español. Estamos de acuerdo con la crítica que se hace de los Principios Orientadores de la AEA por parte de algunos miembros de la comunidad internacional (HENDRICKS y CONNER, 1994). En este artículo se recoge, entre otros, el comentario del Prof. Singh de la India que dice que no sólo no existe asociación profesional de evaluadores en su país sino que los evaluadores, que trabajan como profesionales individuales, están necesariamente más preocupados por el crecimiento de la actividad de evaluación (es decir, que haya y se genere actividad evaluativa sistemática) que en hacer de la evaluación una profesión. De forma similar, en España parece prioritario en este momento que se den situaciones de evaluación, y que los programas y políticas públicas se evalúen. Una vez se vaya generando una mínima práctica y cultura evaluativa, entonces se podrá empezar a pensar en la evaluación como una profesión. Una parte clave de este crecimiento será la creación de programas de formación específicos para futuros evaluadores y gestores de evaluación. Esperemos que los primeros intentos de formación de especialistas vengan acompaña- dos de la aparición de un mínimo mercado de trabajo que pueda generar alguna expectativa profesional y viceversa.

De esta manera, la traducción de estos códigos al contexto español debe hacerse con cierta cautela. Los códigos se generan en un contexio anglosajón. Especiảmente en Norteamérica el volumen de evaluaciones de todo tipo y condición es inmenso y en él hay profesionales que se dedican y viven de evaluar y organizaciones que contratan evaluadores para ello. Por eso, señalamos aquí las dos cuestiones que pueden parecer más lejanas de estos códigos respecto al contexto español. En primer lugar, los códigos están formulados desde la perspectiva del evaluador externo, porque es la práctica más común en el contexto anglosajón. Sin embargo, haciendo la posible atraducción a situaciones de evaluación interna, los códigos siguen resultando útiles. En segundo lugar, las últimas tendencias en evaluación, y esto se refleja también en los códigos, es insistir en aprovechar lo mucho ya investigado y evaluado y fomentar la sintesis de investigaciones, el metanálisis y la metaevaluación ${ }^{8}$. Resulta chocante, si no risible, leer en los manuales norteamericanos lo crucial de seleccionar adecuadamente los estudios que van a formar parte de un metanálisis o una metaevaluación. En España no tenemos problemas con la selección, sino con encontrar algún estudio que pudiera formar parte de este tipo de análisis, ya que no tenemos un gran acervo de estudios de evaluación o de investigación de políticas que podamos sintetizar.

Sin embargo, insistimos en la utilidad de estos códigos siempre que se traduzcan y adapten adecuadamente. Especialmente las Normas del Joint Committee son un recurso excelente para la realización de un repaso de la filosofía, la teoría y la práctica de la evaluación. Es una lástima que la segunda edición de 1994 no esté todavía traducida al castellano, porque es un recurso especialmente valioso, desde nuestro punto de vista, para la formación en temas de evaluación. Con respecto a los Principios Orientadores de la AEA, también puede resultar útil su uso, sobre todo para discutir los problemas éticos de la evaluación y para reconocer y elaborar profesional y académicamente sobre el contexto político en el que se desarrolla cualquier práctica evaluativa. Como dice House (1995, p. 28), "tenemos este tipo de problemas (dilemas éticos) y debemos hablar de ellos, incluso aunque no podamos resolverlosn. Efectivamente, los códigos deontológicos pueden resultar un buen punto de partida para discutir sobre las cuestiones éticas implícitas en la evaluación y para afrontar los dilemas y situaciones problemáticas que puede encontrar cualquier evaluador en su práctica profesional cotidiana. 


\section{Anexo 1: Códigos deontólogicos en evaluación}

NORMAS PARA LA EVALUACIÓN DE PROGRAMAS

The Joint Committee on Standards for Educational Evaluation

\section{Edición. 1994}

"Una norma es un principio en el que se han puesto de acuerdo personas que están comprometidas en la práctica profesional y que, si se cumple, intensificará la calidad e imparcialidad de dicha práctica profesional, por ejemplo, la evaluación".

\section{NORMAS DE UTILIDAD}

Las normas de utilidad pretenden asegurar que una evaluación servirá a las necesidades de información de los usuarios pretendidos.

\section{U1 Identificación de los stakebolders ${ }^{9}$}

Se deben identificar las personas implicadas 0 afectadas por la evaluación, de manera que sus necesidades puedan ser cubiertas.

\section{U2 Credibilidad del evaluador}

Las personas que dirijan una evaluación deben ser merecedoras de confianza y competentes para realizar una evaluación, de manera que los hallazgos de la evaluación consigan la máxima credibilidad y aceptación.

\section{U3 Selección y alcance de la información}

La información recopilada debe ser seleccionada ampliamente para contestar preguntas pertinentes sobre el programa y debe responder a las necesidades e intereses de clientes y otros implicados.

\section{U4 Identificación de valores}

Las perspectivas, los procedimientos y la fundamentación que se utilicen al interpretar los resultados deben describirse con cuidado, de manera que los fundamentos de los juicios de valor sean claros.

\section{U5 Claridad del informe}

Los informes de evaluación deben describir claramente el programa que está siendo evaluado, incluyendo su contexto, y los propósitos, procedimientos y resultados de la evaluación, de manera que se ofrezca información esencial y fácilmente comprensible.

\section{U6 Oportunidad y difusión del informe}

Los hallazgos intermedios significativos y los informes de evaluación se deben difundir a los usuarios deseados, de manera que puedan ser utilizados de manera oportuna.

\section{U7 Impacto de la evaluación}

Las evaluaciones se deben planificar, dirigir y comunicar de manera que animen a los implicados a su cumplimiento, de manera que aumente la probabilidad de que se use la evaluación.

\section{NORMAS DE FACTIBIIDAD/VIABIIDAD}

Las normas de factibilidad tienen el propósito de asegurar que una evaluación sea realista, prudente, diplomática y frugal.

\section{F1 Procedimientos prácticos}

Los procedimientos de la evaluación deben ser prácticos, de manera que las interrupciones sean mínimas mientras se obtiene la información necesaria.

\section{F2 Viabilidad politica}

La evaluación se debe planificar y dirigir anticipando las diferentes posturas de diversos grupos de interés, de manera que se pueda obtener su colaboración y así se puedan advertir y contrarrestar posibles intentos de restringir las operaciones de evaluación o de sesgar o abusar de los resultados por parte de estos grupos.

\section{F3 Coste Efectividad}

La evaluación debe ser eficiente y producir una información de valor suficiente, de manera que se justifiquen los recursos empleados.

\section{NORMAS DE CORRECCIÓN /LEGITIMIDAD ${ }^{10}$}

Son las normas dirigidas a asegurar que la evaluación se dirija legal y éticamente y con la debida atención al bienestar de aquellos implicados en la evaluación, así como a aquellos afectados por sus resultados.

\section{C1 Orientación de Servicio}

Las evaluaciones se deben diseñar para asistir a las organizaciones a tratar y cubrir efectivamente las necesidades de todo el espectro de destinatarios.

\section{C2 Acuerdos formales}

Las obligaciones de las partes formales de la evaluación (qué se hará, cómo, por quién y cuándo) se deben acordar por escrito, de forma que que dichas partes estén obligadas a seguir todas las condiciones del acuerdo o, por el contrario, renegociarlo formalmente. 


\section{C3 Derechos de los sujetos}

Las evaluaciones se deben diseñar y dirigir de tal forma que se respeten y protejan los derechos y el bienestar de los sujetos.

\section{C4 Interacciones humanas}

Los evaluadores deben respetar la dignidad y el valor humano en sus interacciones con otras personas asociadas a la evaluación, de manera que no se amenace o dañe a los participantes.

\section{C5 Valoración completa y justa}

La evaluación debe ser completa y justa en su análisis y en su registro de fortalezas y debilidades del programa evaluado, de tal modo que las fortalezas sean reforzadas y las áreas problemáticas tratadas.

\section{C6 Revelación de los resultados}

Las partes formales de la evaluación deben asegurar que el conjunto completo de resultados de la evaluación junto con sus pertinentes limitaciones son accesibles para las personas afectadas por la evaluación y para cualquier otra persona con derechos legales expresados a recibir los resultados.

\section{C7 Conflicto de intereses}

Los conflictos de intereses deben manejarse abierta y honestamente de tal forma que no comprometan el proceso ni los resultados de la evaluación.

\section{C8 Responsabilidad fiscal}

La distribución y los gastos de recursos asignados por el evaluador deben reflejar procedimientos solventes de información y responsabilidad y si no el evaluador debe ser prudente y éticamente responsable de manera que los gastos se justifique y sean apropiados.

\section{NORMAS DE PRECISIÓN}

Estas normas tienen el propósito de asegurar que una evaluación revele y proporcione información técnicamente apropiada sobre las características que determinan el valor o mérito del programa evaluado.

\section{P1 Documentación del programa}

El programa evaluado debe ser descrito y documentado de forma apropiada y clara, de tal manera que el programa se identifique claramente.

\section{P2 Análisis del contexto}

El contexto en el cual el programa existe se debe examinar con suficiente detalle de manera que se puedan identificar sus posibles influencias sobre dicho programa.

\section{P3 Descripción de propósitos y procedimientos}

Los propósitos y procedimientos de la evaluación debẹn ser seguidos y descritos con el suficiente detalle de manera que se puedan identificar y valorar.

\section{P4 Fuentes de información defendibles}

Las fuentes de información que se utilicen en una evaluación de programa se deben describir con suficiente detalle de forma que se pueda estimar la adecuación de la información.

\section{P5 Información válida}

Los procedimientos de recopilación de información se deben elegir y desarrollar y entonces poner en práctica de manera que se asegure que la interpretación a la que se ha llegado es válida para el uso que se le pretende dar.

\section{P6 Información fiable}

Los procedimientos de recopilación de información se deben elegir y desarrollar y entonces poner en práctica de manera que se asegure que la información obtenida es suficientemente fiable para el uso que se le pretende dar.

\section{p7 Información sistemática}

La información recopilada, procesada y trasmitida en una evaluación debe ser revisada sistemáticamente y cualquier error encontrado debe ser corregido.

\section{P8 Análisis de la información cuantitativa}

La información cuantitativa de una evaluación se debe analizar apropiada y sistemáticamente de manera que las preguntas de evaluación se contesten de forma efectiva.

\section{P9 Análisis de la información cualitativa}

La información cualitativa de una evaluación se debe analizar apropiada y sistemáticamente de manera que las preguntas de evaluación se contesten de forma efectiva.

\section{P10 Conclusiones justificadas}

Las conclusiones a las que se llega en una evaluación deben estar justificadas explícitamente de manera que los implicados y afectados por la evaluación puedan ponderarlas.

\section{P11 Informe imparcial}

Los procedimientos para informar deben asegurar que no haya distorsión causada por sesgos y sentimientos personales de cualquiera de las pärtes de la evaluación, de manera que los informes reflejen de forma justa los resultados de la evaluación.

\section{P12 Metaevaluación}

La evaluación en sí misma se debe evaluar formativa y sumativamente a través de éstas y otras normas pertinentes, de manera que se guíe de forma apropiada su realización y, 
en cuanto se termine, los implicados y afectados puedan examinar de cerca sus fortalezas y debilidades.

\section{PRINCIPIOS ORIIENTADORES PARA EVALUADORES (Re- sumen)}

\section{(Asociación Americana de Evaluación, 1994)}

\section{Premisas relacionadas con el desarrollo de los principios}

1. Estos principios se dirigen a todo tipo de evaluaciones (de programas, de productos, de personal, de políticas, del desempeño, de propuestas, de tecnologías, de la investigación, de teorías y de la propia evaluación).

2. A pesar de la diversidad respecto a los propósitos de la evaluación, lo común es que los evaluadores aspiren a construir y proporcionar la mejor información posible que se pueda aportar sobre el valor de lo que se evalúe.

3. La intención es guiar la práctica profesional de los evaluadores. No son sólo una guía para la discusión; estos principios deben guiar proactivamente los comportamientos de los profesionales en la práctica cotidiana.

4. El propósito de documentar los principios es el de fomentar el desarrollo continuo de la profesión de evaluadores, así como la socialización de sus miembros. Pretenden estimular la discusión.

5. Los cinco principios no son independientes sino que se solapan de muchas maneras. De manera inversa, a veces, estos principios entrarán en conflicto entre sí, haciendo que los evaluadores tengan que elegir entre los mismos.

6. Estos principios son la postura oficial de la AEA.

7. Las declaraciones en cada principio están concebidas para ampliar el significado de los mismos y aportar orientaciones para su aplicación. Ni pretenden incluir todas las posibles aplicaciones del principio ni deben ser vistas como reglas que provean de una base para sancionar evaluadores.

8. No pretenden sustituir otras normas de otras disciplinas. AEA apoya al Joint Committee, del cual es copatrocinador.

9. Estos principios se han desarrollado en el contexto de la cultura occidental, y más concretamente en los Estados Unidos. Su relevancia puede variar en otras culturas.

10. Estos. principios son parte de un proceso en evolución de auto-examen de la profesión y deben ser revisados periódicamente, al menos cada 5 años.

\section{A. Indagación sistemática}

Los evaluadores dirigen indagaciones sistemáticas y basadas en datos, sobre cualquier cosa que se evalúe. Deben:
1. Observar las normas técnicas más apropiadas.

2. Estudiar las deficiencias y fortalezas de las posibles preguntas y aproximaciones.

3. Comunicar los métodos y enfoques utilizados y dejar claras las limitaciones de la evaluación y sus resultados.

\section{B. Competencia}

Los evaluadores proporcionan una actuación competente a los implicados en la evaluación (stakebolders). Deben:

1. Poseer la educación, capacidades, habilidades y experiencia apropiadas.

2. Ejercer dentro de los límites de su formación y competencia profesional y deben declinar dirigir evaluaciones que excedan dichos límites.

3. Procurar mantener y mejorar sus competencias de forma continuada.

\section{Integridad/Honestidad}

Los evaluadores aseguran la honestidad e integridad de todo el proceso de evaluación. Deben:

1. Negociar honestamente con los clientes y las personas implicadas más relevantes, los costes, las tareas a realizar, las limitaciones metodológicas, el posible alcance de los resultados y la utilización de dichos resultados.

2. Anotar e informar sobre los cambios hechos sobre el proyecto de evaluación negociado, y las razones para el cambio.

3. Determinar los intereses respecto a la realización y los efectos de la evaluación (propios, del cliente y otros implicados).

4. Revelar cualquier rol o relación que pueda producir un conflicto de interés significativo con su rol como evaluador.

5. No deben tergiversar los procedimientos, los datos o los resultados.

6. Si los evaluadores determinan que ciertos procedimientos o actividades tienen probabilidad de producir información evaluativa o conclusiones erróneas, tienen la responsabilidad de comunicar sus preocupaciones y sus razones al cliente.

7. Salvo que haya una razón convincente para lo contrario, los evaluadores deben revelar todas las fuentes de financiación de la evaluación, así como de dónde ha venido el requerimiento de la evaluación. 


\section{Respeto por las personas}

Los evaluadores respetan la seguridad, dignidad y valor personal de los informantes, participantes en el programa, clientes y otros implicados y afectados con los que interacrúan. Deben:

1. Atenerse a la ética profesional y a las normas establecidas en lo que respecta a los riesgos, daños y en lo que respecta al consentimiento de participar en la evaluación.

2. Tratar de maximizar los beneficios y reducir cualquier daño innecesario que pueda darse, teniendo en cuenta que esto no comprometa la integridad de los resultados de la evaluación.

3. Realizar la evaluación y comunicar sus resultados de forma que se respete claramente la dignidad y el valor personal de dichos implicados.

4. Intentar promover la igualdad social de la evaluación, de manera que aquellos que han dado a la evaluación puedan obtener algunos beneficios a cambio.

5. Indentificar y respetar las diferencias entre los participantes (culturales, religiosas, de género, de capacidad, de edad, de orientación sexual y étnicas) y ser conscientes de las implicaciones potenciales de estas diferencias mientras se planifi$\mathrm{ca}$, dirige, analiza e informa sobre sus evaluaciones.

\section{E. Responsabilidades bacia el bienestar público y general}

Los evaluadores articulan y tienen en cuenta la diversidad de intereses y valores que pueden estar relacionados con el bienestar público y general. Deben:

1. Considerar la inclusión de las perspectivas e intereses de todos los implicados en el programa u objeto evaluado.

2. Considerar no sólo los resultados inmediatos, sino también las premisas subyacentes, las implicaciones y los efectos laterales potenciales.

3. Permitir a todos los implicados relevantes acceso a la información evaluativa y deben proporcionársela si los recursos lo permiten. Si se comunican diferentes resultados de evaluación en formas diseñadas específicamente a determinados implicados, los otros grupos de implicados deben conocer su existencia. Deben presentar los resultados tan clara y simplemente como la precisión lo permita.

4. Mantener un equilibrio entre las necesidades de los clientes y otras necesidades. También deben luchar por cubrir las necesidades legítimas de los clientes siempre que sea posible y apropiado. En caso de conflicto, deben discutirlo explícitamente con el cliente y los implicados relevantes.

5. Lograr el interés y el bien público. Estas obligaciones son especialmente importantes cuando los evaluadores están sos- tenidos por fondos públicos. Para esto, tendrán que ir más allá del análisis de los intereses de un implicado particular, considerando el bienestar de la sociedad en general.

\section{ORIENTACIONES PARA UNA ACTUACIÓN ÉTICA}

\section{(Sociedad Canadiense de Evaluación, 1996)}

\section{Competencia}

Los evaluadores deben ser competentes en el suministro de sus servicios. Para ello deben:

1.1. Aplicar métodos sistemáticos de indagación apropiados a la evaluación.

1.2. Poseer o proporcionar conocimiento apropiado para la evaluación.

1.3. Luchar de forma continuada para mejorar sus habilidades prácticas y metodológicas.

\section{Integridad}

Los evaluadores deben actuar con integridad al relacionarse con todos los implicados (stakebolders) en la evaluación. Para ello deben:

2.1. Representar correctamente su nivel de conocimiento y habilidades.

2.2. Declarar a los clientes cualquier conflicto de intereses antes de embarcarse en un proyecto de evaluación y en cualquier punto en el que dicho conflicto pueda ocurrir. Esto incluye los conflictos de intereses por parte de los implicados y del evaluador.

2.3. Ser sensibles ante el ambiente social y cultural de todos los implicados y actuar de forma apropiada ante dicho ambiente.

2.4. Debatir con el cliente decisiones contractuales tales como: confidencialidad, privacidad, comunicación y propiedad de los resultados y los informes.

\section{Responsabilidad (Accountability)}

Los evaluadores deben hacerse responsables de su' desempeño y su producto. Para ello deben ser responsables de:

3.1. Ofrecer la información necesaria a los clientes para que puedan tomar decisiones sobre cuáles son las metodologías y estrategias apropiadas para la evaluación. Dicha información debe incluir las limitaciones de la metodología elegida. 
3.2. Ofrecer una presentación escrita y oral, que sea clara, correcta y justa, de los resultados y las limitaciones del estudio, así como de las recomendaciones.

3.3. Sus decisiones fiscales, de manera que se rinda cuenta de los gastos de la evaluación y los clientes reciban un buen producto por su dinero.

3.4. Completar la evaluación en un tiempo razonable y dentro de lo acordado con los clientes. Tales acuerdos deben tener en cuenta anteriores retrasos debidos a factores más allá del control del evaluador.

\section{ORIENTACIONES PARA UNA ACTUACIÓN ÉTICA EN EVALUACIONES (Resumen)}

\section{(Sociedad Australasiática de Evaluación, 1997)}

\section{A. Encargo y preparación de una evaluación}

Principios:

- Todas las partes implicadas en el encargo y dirección de una evaluación deben estar totalmente informadas sobre lo que se espera y razonablemente se ofrecerá, de forma que puedan sopesar los riesgos éticos antes de firmar el acuerdo.

- Todas las personas que puedan ser afectadas si se procede o por cómo se proceda en una evaluación deben tener la oportunidad de identificar formas en las que los riesgos se puedan reducir.

Orientaciones. Se debe:

1. Preparar un breve documento/términos de referencia que refleje: fundamentación, propósito, alcance, cuestiones claves y destinatarios de la evaluación.

2. Identificar limitaciones metodológicas y éticas e intereses distintos.

3. Acordar un contrato que especifique: condiciones, recursos, servicios a proporcionar, precio a pagar, calendario de entrega, propiedad de los datos e informes, protección y almacenamiento de información, procedimientos para dirimir posibles disputas, papel del cliente en la revisión de informes, emisión y publicación de informes y posibles usos de los materiales de la evaluación.

4. Avisar sobre circunstancias cambiantes en el acuerdo (por parte de clientes y evaluadores).

5. Tener en cuenta los riesgos o daños potenciales y negociarlos inicialmente.

6. El equipo de evaluación debe poseer el concimiento, capacidades, habilidades y experiencia necesaria para llevar a cabo la evaluación.
7. Revelar e identificar cualquier conflicto potencial de intereses.

8. Competir honorable y profesionalmente.

9. Las personas que encargan una evaluación y/o seleccionan un evaluador deben tratar las propuestas de forma justa y abierta, respetando la propiedad intelectual y de los materiales y la confidencialidad comercial.

\section{B. Realización de una evaluación}

Principios:

- La evaluación se debe diseñar, dirigir y comunicar de manera que se respeten los derechos, la privacidad y la dignidad de las personas afectadas por la evaluación y las que contribuyen a la misma.

- Una evaluación se debe realizar de manera que se asegure que los enjuiciamientos que se realizan son resultado de la evaluación y que cualquier acción relacionada con ella se basa en una información completa y sólida.

Orientaciones. Se debe:

10. Considerar las implicaciones de diferencias y desigualdades, relacionadas con raza, edad, género, orientación sexual, capacidad intelectual o física, religión, bagaje socio-económico, cultural y étnico.

11. Los evaluadores se deben identificar y avisar sobre su propósito.

12. Obtener consentimiento de las personas de las que se obtiene información (preferiblemente por escrito).

13. La evaluación debe ser rigurosa en el diseño, la recopilación de información y el análisis.

14. Declarar las limitaciones encontradas.

15. Mantener la confidencialidad durante el proceso de evaluación.

16. Informar sobre cualquier problema significativo inesperado que se encuentre en el curso de la evaluación.

17. Cuando los evaluadores descubran alguna actividad criminal potencial o real deben responder ante sus responsabilidades éticas y legales. Éstos deben:

* evitar o reducir mayores daños a las víctimas,

* cumplir con sus obligaciones legales y de sus códigos éticos profesionales que pueden incluir informar a la autoridad competente,

* mantener los acuerdos realizados con los informantes respecto a la confidencialidad. 


\section{Comunicación de los resultados de una evaluación}

Principio:

- La evaluación se debe comunicar de tal manera que los destinatarios obtengan una respuesta imparcial y equilibrada a los términos de referencia de la evaluación.

Orientaciones. Se debe:

18. Presentar los resultados de forma clara y simple.
19. Los informes deben ser directos, completos y honestos, incluyendo suficientes detalles de la metodología y de los resultados para apoyar las conclusiones.

20. Identificar las fuentes y reconocer a aqueilas personas que han contribuido.

21. El informe final debe reflejar en su totalidad los resultados y las conclusiones determinadas por el evaluador.

22. Al divulgar la información basada en los informes de evaluación, los clientes tienen las responsabilidad de no violar la integridad de los mismos.

\begin{abstract}
Universidad Complutense de Madrid.
${ }^{1}$ Entre otras se pueden mencionar Evaluation Practice (recientemente convertida en The American Journal of Evaluation), Evaluation Review, Evaluation and Programa Planning, Educational Evaluation and Policy Analysis, Evaluation and the Health Professions, New Directions for Programa Evaluation y la desaparecida Evaluation Studies Review Annual.

2 En este artículo se incluyen, en el anexo, resúmenes de los cuatro códigos existentes: el del Joint Committee y los elaborados y aprobados por tres asociaciones profesionales: la Asociación Americana de Evaluación (AEA), la Sociedad Canadiense de Evaluación (CES) y la Sociedad Australasiática de Evaluación (AES). Para consultar los documentos originales promovidos por las tres asociaciones se pueden visitar sus respectivas aweb page:

American Evaluation Assotiation <http://www.eval.org>

Australasian Evaluation Society <http://www.parklane.com.au/aes/ethics.html>

Canadian Evaluation Society <http://www.unites.uqam.ca/sce/ethicse.html>

3 Como se verá más adelante, esta generalidad es más achacable a los Principios Orientadores de la AEA que a las Normas del Joint Committee.

${ }^{4}$ Ya hay muchas profesiones en EE.UU. que, para ejercerlas, sus profesionales deben estar convenientemente acreditados. Esto supone no sólo estar colegiado (como es el caso español con los médicos, psicólogos, arquitectos y abogados) sino pasar un examen que debe ser renovado cada cierto tiempo y convalidado en los territorios en los que se va a ejercer (cada Estado suele tener sus reglas de acreditación).

5 Este comité, compuesto por representantes de la Asociación Americana de Investigación Educativa (AERA), la Asociación Americana de Psicología (APA) y el Consejo Nacional para la Medición Educativa (National Council on Measurement
\end{abstract}

inEducation), se reúne en el año 1974, teniendo como cometido la revisión de las Normas para Manuales y Tests Psicológicos y Educativos publicados por la APA en 1966.

- El cerebro que le falta al espantapájaros sería necesario para los principios de indagación sistemática y competencia. El corazón que le falta al hombre de hojalata estaría relacionado con los principios de respeto por la gente y responsabilidad por el bienestar público y general. El valor que le falta al león cobarde se puede asociar con el principio de integridad y honestidad (T.D. KNoTT, 1998).

' Por su interés, aparecen también traducidas y resumidas junto con los principios orientadores.

${ }^{8}$ El metanálisis es una técnica muy sofisticada para analizar conjuntamente diferentes investigaciones sobre una misma materia. la metaevaluación se suele utilizar en dos sentidos: en primer lugar, evaluar procesos evaluativos -es decir, evaluar cómo se han realizado las evaluaciones- y también como evaluación de un determinado campo a partir de los resultados de diferentes evaluaciones.

${ }^{9}$ Constatamos aquí la dificultad de traducción al castellano del término stakebolder, que vendría a ser algo así como el que tiene algo que decir o tiene algún interés en. Según el Joint Committee la definición de stakebolder en evaluación sería Jos individuos o grupos que pueden estar implicados en o afectados por una evaluación. Aunque sería más correcto traducirlos como implicados en y afectados por la evaluación, por razones de economía lingüística traducimos stakebolder como implicados en la evaluación.

10 El término original en inglés es propriety standars. Aunque nos parece más adecuada la traducción de normas de corrección, mantenemos también el término de legitimidad. que es el que aparece en la traducción al castellano de la 1. a edición de las normas (México, Ed. Trillas, 1988).

\section{Bibliografia}

ALTSCHULD, J.W. y ENGLE, M. (eds.) (1994), The Preparation of Professional Evaluators: Issues, Perspectives and Programs. New Directions for Program Evaluation, n. ${ }^{9} 62$, San Francisco: Jossey-Bass.

CHELIMSKY, E. (1995), .Comments on the Guiding Principles. En W.R. SHADish et al. (eds.) Guiding Principles for Evaluators. New Directions for Program Evaluation, n. ${ }^{\circ}$ 66, San Francisco: Jossey-Bass, pp. 35-54.

HendRICKS, M. y ConNer, R.F. (1995), -Intemational Perspectives on the Guiding Principles. En W.R. SHADISH et al. (eds.), Guiding Principles for Evaluators. New Directions for Program Evaluation, n. 66 , San Francisco: Jossey-Bass, pp. 77-90.
HousE, E.R. (1995), Principles Evaluation: A Critique of the AEA Guiding Principles. En W.R. SHADISH et al. (eds.), Guiding Principles for Evaluators. New Directions for Program Evaluation, n. ${ }^{2}$ 66, San Francisco: Jossey-Bass, pp. 27-34.

Joint Committee on Standards for Educational Evaluation (1988), Normas de evaluación para programas, proyectos y material educativo. México: Trillas.

(1994), The Program Evaluation Standards. $2^{\text {nd }}$ Edition. Thousand Oaks: Sage. 
KvotT, T.D. (1998) •A Wiz of a Way to Remember the Five Guiding Principles for Evaluators. American Journal of Evaluation, vol. 19, n. ${ }^{\circ} 1$, pp 135-139.

Rossi, P.H. (1995), ‘Doing It Good and Getting It Rights. En W.R. SHADISH et al. (eds.), Guiding Principles for Evaluators. New Directions for Program Evaluation, n.. 66, San Francisco: Jossey-Bass, pp. 55-60.
SANDERS, J.R. (1995), Standards and Principles*. En W.R. SHADISH et al. (eds.), Guiding Principles for Evaluators. New Directions for Program Evaluation, n. 66 , San Francisco: Jossey-Bass, pp. 47-52

SHADISH, W.R. et al. (eds.) (1995), Guiding Principles for Evaluators. New Directions for Program Evaluation, n.․ 66, San Francisco: Jossey-Bass. 